KANSAS JOURNAL of MEDICINE

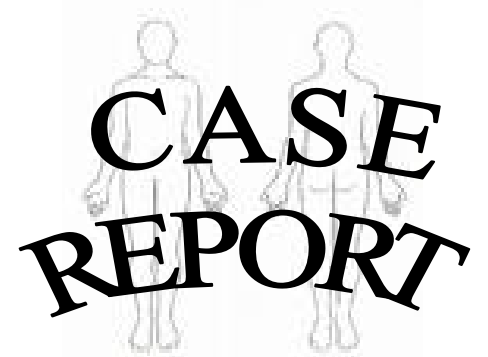

\section{Gastrointestinal Stromal Tumor Found Incidentally in a Patient with Multiple Endocrine Neoplasia Type I (MENI)}

Rossa Khalaf, M.D. ${ }^{1}$, Rami Mortada, M.D. ${ }^{2}$ ${ }^{1}$ Banner University Medical Center, Phoenix, AZ ${ }^{2}$ Univeristy of Kansas School of Medicine-Wichita Department of Internal Medicine

\section{INTRODUCTION}

Gastrointestinal stromal tumor (GIST) is a rare tumor arising from the mesenchymal tissue of the sub-epithelial mucosal surface of the gastrointestinal tract. ${ }^{1}$ It is mainly located in the stomach and proximal intestine, but can be seen in other parts of the gastrointestinal tract. It is characterized by expression of CD117 which is a part of the C-kit proto-oncogene product (KIT) transmembrane receptor tyrosine kinase in more than $80 \%$ of cases. ${ }^{2}$ Some cases express a mutation in PDGFRA (platelet-derived growth factor receptor, alpha polypeptide). GIST has the potential to become a malignant tumor, which can metastasize to the liver and peritoneum. ${ }^{3-5}$

Multiple endocrine neoplasia type 1 (MEN1) syndrome is an autosomal dominant (AD) disorder, characterized by inactivation of the Menin gene, a tumor suppressor located on the long arm of chromosome 13 (13q11). ${ }^{6}$ MEN1 syndrome is associated with pituitary, parathyroid, and pancreatic islet tumors. ${ }^{7,8}$ Some non-endocrine benign tumors also are associated with MEN1 syndrome, such as facial angiofibromas, lipomas, meningioma, and leiomyoma. ${ }^{9}$ The association between GIST tumors and MEN1 syndrome, however, is unclear. To our knowledge, only five cases had reported GIST tumors in association with MEN1 syndrome. ${ }^{10-12}$

\section{CASE REPORT}

A 60 -year-old male had a $6 \mathrm{~cm}$ mass of the pancreas body and tail discovered on a computed tomography (CT) scan of the abdomen which was done to evaluate recurrent hypercalcemia nephrolithiasis. The diagnosis of a well-differentiated neuroendocrine pancreatic tumor was made by fine needle aspiration (FNA). Following staging, surgical resection was performed (T3NOM0). In addition to that, another $2 \mathrm{~cm}$ tumor was attached to the distal body and along the greater curvature of the stomach. Pathology of this new tumor revealed a GIST tumor with positive C-kit proto-oncogene product (CD117) and negative S-100 calcium binding protein.

At the time of surgery, the patient's serum calcium was elevated up to $11.4 \mathrm{mg} / \mathrm{dl}$. His parathyroid hormone was $53 \mathrm{pg} /$ $\mathrm{ml}$ and spot urine calcium was $28.4 \mathrm{mg} / \mathrm{dl}$. Pituitary function testing, including levels of follicle-stimulating hormone, thyroid-stimulating hormone, prolactin, insulin-like growth factor, and adrenocorticotropic hormone were within normal range with the exception of a mild elevation of luteinizing hormone. A Sestamibi scan revealed persistent radiotracer uptake in the region of the right inferior parathyroid gland thought to be consistent with a parathyroid adenoma. Magnetic resonance imaging (MRI) of the brain revealed a $0.5 \mathrm{~cm}$ pituitary adenoma.

The patient underwent parathyroidectomy. As a result, the diagnosis of MEN1 was made clinically, although a genetic test for MEN1 was negative. As the patient was recovering, another CT scan of the abdomen was taken to investigate the reason of his persistent hematuria and nephrolithiasis. This scan revealed a new $1.9 \mathrm{~cm}$, peripherally enhancing mass on the ventral side of the pancreas head. Endoscopic ultrasound (EUS) revealed another neuroendocrine tumor of the pancreas. At that point, the patient underwent exploratory laparotomy for resection of this neuroendocrine tumor with no residual metastasis. He recovered well after the surgery. His following labs revealed normal calcium as well as vitamin D level.

\section{DISCUSSION}

This case of MEN1 syndrome was associated with GIST of the stomach and found incidentally during a surgical removal of a pancreatic neuroendocrine tumor. GIST is the most common nonepithelial tumor of the gastrointestinal tract and may become malignant in $1 \%$ of cases. ${ }^{3-5,13,14}$ Usually GIST tumors are asymptomatic and discovered incidentally on imaging or endoscopy. However, some patients may experience non-specific symptoms such as early satiety, bloating, and ulceration with bleeding. ${ }^{1}$

Diagnosis of MEN1 is usually established by one of the following three criteria: 9,15

1. Having two or more of the classical primary three tumors: parathyroid gland, anterior pituitary, or enter-pancreatic cell (such as gastrinoma, insulinoma, vasoactive intestinal polypeptide secreting tumor, and non-hormone secreting).

2. Determination of DNA genetic mutation in an individual who does not have clinical or biochemical features.

3. Having a family member with MEN1 syndrome. DNA testing is completed to identify any menin mutation at chromosome 11q13 which is found in up to $90 \%$ of the cases. ${ }^{79}$ Usually DNA testing is offered to any index patient with clinical MEN1 (two or more primary MEN1 tumor types), all firstdegree relatives of known MEN1 mutation carriers, and to individuals with suspicious or atypical MEN1 (e.g., multiple parathyroid adenomas, gastrinoma, or multiple pancreatic neuroendocrine tumors). ${ }^{7}$ In our case, the diagnosis was based on the clinical findings of the classical tumors of MEN1 syndrome.

In addition to the classical findings, non-endocrine tumors have been identified in some MEN1 cases such as carcinoid tumors, 


\section{KANSAS JOURNAL of MEDICINE}

GASTROINTESTINAL STOMALTUMOR

continued.

adrenocortical tumors, meningioma, facial angio-fibromas, and lipomas. ${ }^{9}$ The MEN1 gene was suggested to act as a recessive tumor suppressor gene due to high rate of heterozygosity which was detected in the primary tumors as well as non-classical tumors. ${ }^{8,16}$

Few previous case reports detected GIST in association with MEN1 syndrome. None detected any loss of heterozygosity (LOH). ${ }^{10-12}$ Another case report was unable to detect any LOH in 11q13 area in a MEN1 syndrome associated with GIST using the PYGM microsatellite polymorphism and a MEN-1 intragenic polymorphism localized in exon 9 (D418D). ${ }^{17}$ Different mechanisms were suggested to explain this association: (1) an unknown independent mechanism or (2) a loss of the genes located closely to 11q13 gene.

The etiology of our patient's hypercalcemia was likely secondary to hyperparathyroidism as his calcium level trended down after parathyroidectomy. Hypercalcemia has been associated with GIST tumor. ${ }^{18}$ The etiology was thought to be due to PTHrelated peptide production. However, one recent case reported hypercalcemia associated with GIST tumor in the setting of suppressed PTH and ProPTHbutelevated 1,25(OH)2 vitamin Dlevel.

\section{CONCLUSION}

We reported another case in which MEN1 was associated with GIST. Yet, no evidence-based data support or explain the clinical association between GIST and MEN1 syndrome. However, this association should be considered when a GI tumor is associated with clinical features of MEN1 syndrome. Hypercalcemia likely was secondary to hyperparathyroidism. Although GIST induced hypercalcemia caused by converting vitamin $\mathrm{D}$ to its active form also should be considered.

\section{REFERENCES}

1 DeMatteo RP, Lewis JJ, Leung D, Mudan SS, Woodruff JM, Brennan MF. Two hundred gastrointestinal stromal tumors: Recurrence patterns and prognostic factors for survival. Ann Surg 2000; 231(1):51-58. PMID: 10636102. 2 Hirota S, Ohashi A, Nishida T, et al. Gain-of-function mutations of platelet-derived growth factor receptor alpha gene in gastrointestinal stromal tumors. Gastroenterology 2003;125(3):660-607.PMID:12949711.

3 Antonioli DA. Gastrointestinal autonomic nerve tumors. Expanding the spectrum of gastrointestinal stromal tumors. Arch Pathol Lab Med 1989; 113(8):831-833. PMID: 2474285. 4 Lee JS, Nascimento AG, Farnell MB, Carney JA, Harmsen WS, Ilstrup DM. Epithelioid gastric stromal tumors (leimyoblastomas): A study of fifty-five cases. Surgery 1995; 118(4):653-660. PMID: 7570319. Ballarini C, Intra $\mathrm{M}$, Ceretti $\mathrm{AP}$, et al. Gastrointestinal stromal tumors: A "benign" tumor with hepatic metastasis after 11 years. Turmori 1998; 84(1):78-81. PMID: 9619721. 6 Lemos MC, Thakker RV. Multiple endocrine neoplasia type 1 (MEN1): Analysis of 1336 mutations reported in the first decade of following identification of the gene. Hum Mutat 2008; 29(1):22-32. PMID: 17879353. 7 Thakker RV, Newey PJ, Walls GV, et al. Clinical practice guidelines for multiple endocrine neoplasia type 1 (MEN1). J Clin Endocrinol Metab 2012; 97(9):2990-3011. PMID: 22723327. Friedman E, Sakaguchi K, Bale AE, et al. Clonality of parathyroid tumors in familial multiple endocrine neoplasia type 1. N Engl J Med 1989; 321(4):213-218. PMID: 2568586.
Brandi ML, Gagel RF, Angeli A, et al. Guidelines for diagnosis and therapy of MEN type 1 and type 2. J Clin Endocrinol Metab 2001; 86(12):5658-5671. PMID: 11739416. 10 Dong Q, Debelenko LV, Chandrasekharappa SC, et al. Loss of heterozygosity at 11q13: Analysis of pituitary tumors, lung carcinoids, lipomas, and other uncommon tumors in subjects with familial multiple endocrine neoplasia type 1 . J Clin Endocrinol Metab 1997; 82(5):1416-1420. PMID: 9141526. 11 Burton JL, Martog M. Multiple endocrine adenomatosis (Type 1) with cutaneous leiomyomata and cysts of Moll. Br J Dermatol 1977; 97(Suppl 14):74-75. PMID: 18161. 12 Larraza-Hernandez O, Albores-Saavedra J, Benavides G, Krause LG,Perez-MerizaldiJC,GinzoA.Multipleendocrinemeoplasia.Pituitary adenoma, multicentric papillary thyroid carcinoma, bilateral carotid body paraganglioma, parathyroid hyperplasia, gastric leiomyoma, and systemamyloidosis.Am JClinPathol1982;78(4):527-532. PMID:7137085. ${ }_{13}$ Rubin BP, Fletcher JA, Fletcher CD. Molecular insights into the histogensis and pathogenesis of gastrointestinal stromal tumors. Int J Surg Pathol 2000; 8(1):5-10. PMID: 11493959. 14 Medeiros F, Corless CL, Duensing A, et al. KIT-negative gastrointestinal stromal tumors: Proof of concept and therapeutic implications. Am J Surg Pathol 2004; 28(7):889-894. PMID: 15223958. $15 \quad$ NeweyPJ,ThakkerRV.Roleofmultipleendocrineneoplasiatype1 mutationalanalysisinclinical practice.EndocrPract2011;17(Suppl3):8-17. PMID: 21454234.

16 Radford DM, Ashley SW, Wells SA Jr, Gerhard DS. Loss of heterozygosity of markers on chromosome 11 in tumors from patients with multiple endocrine neoplasia syndrome type 1. Cancer Res 1990; 59(20):6529-6533. PMID: 1976436. 17 Papillon E, Roalchon A, Calender A, Chabre O, Barnoud R, Fournet J. A malignant gastrointestinal stromal tumour in a patient with multiple endocrine neoplasia type 1. Eur J Gastroenterol Hepatol 2001; 13(2):207-211. PMID: 11246625.

18 Jasti P, Lakhani VT,Woodworth A, Dahir KM. Hypercalcemia secondary to gastrointestinal stromal tumors: Parathyroid hormone-related protein independent mechanism? Endocr Pract 2013; 19(6):e158-e162. PMID: 24013983.

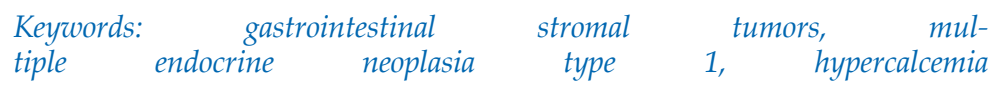

